doi: $\underline{\text { http://dx.doi.org/10.5892/RUVRV.91.7888 }}$

\title{
A FARMACOEPIDEMIOLOGIA NO BRASIL: estado da arte da produção científica
}

\author{
André de Oliveira BALDONI MSc ${ }^{1 *}$ \\ Camilo Molino GUIDONI MSc ${ }^{1}$ \\ Leonardo Régis Leira PEREIRA PhD ${ }^{1}$
}

RESUMO: Introdução. Os estudos farmacoepidemiológicos fornecem informações essenciais para a gestão e utilização dos medicamentos. Objetivo. Estimar quantitativamente os artigos sobre Farmacoepidemiologia publicados mundialmente e analisar o perfil e a evolução dos estudos farmacoepidemiológicos no Brasil. Casuística e Métodos. Foi realizado uma busca através do cruzamento do descritor "Pharmacoepidemiology" e país de interesse dos artigos indexados no PubMed/MedLine. Para busca de artigos brasileiros no SciELO utilizou-se o descritor "Farmacoepidemiologia". A pesquisa em ambas as bases de dados incluiu todos os artigos publicados até 18/06/2011, excluindo-se os de revisão. Resultados e Discussão. Foram encontrados 620 artigos científicos indexados no PubMed/MedLine, destacando-se o maior número de publicações nos Estados Unidos ( $\mathrm{n}=146)$ e no Brasil $(\mathrm{n}=14)$ entre os países desenvolvidos e emergentes selecionados, respectivamente. Com relação aos estudos brasileiros, foram analisados 48 artigos científicos disponíveis no PubMed/MedLine e SciELO. As publicações brasileiras sobre esta temática iniciaram no ano de 2001 e não apresentaram padrão de evolução nas duas bases de dados analisadas, com concentração dos artigos nas regiões Sudeste e Sul do país $(n=40)$. Houve prevalência da utilização de fontes primárias $(n=33)$ para obtenção das informações e discrepância na distribuição dos artigos entre as três vertentes da Farmacoepidemiologia, com predomínio dos Estudos de Utilização de Medicamentos (n=39). Conclusão. Apesar do discreto crescimento no número de artigos sobre Farmacoepidemiologia no Brasil, existe uma discrepância quantitativa quando comparado aos realizados nos países da Europa e América do Norte. Além disso, existe a necessidade de se realizar estudos farmacoepidemiológicos de abrangência nacional e em diferentes grupos populacionais.

Palavras-chave: Brasil. Estudos de Utilização de Medicamentos. Farmacoepidemiologia. Farmacoeconomia. Farmacovigilância.

ABSTRACT: Introduction. Pharmacoepidemiological studies provide essential information for the management and use of drugs. Objective. To estimate the number of the articles published worldwide in the field of Pharmacoepidemiology and to analyze the profile and evolution of pharmacoepidemiological studies in Brazil.

${ }^{1}$ Centro de Pesquisa em Assistência Farmacêutica e Farmácia Clínica (CPAFF), Departamento de Ciências

Farmacêuticas, Faculdade de Ciências Farmacêuticas de Ribeirão Preto, Universidade de São Paulo, Brasil .

*Correspondência: MSc André de Oliveira Baldoni, Departamento de Ciências Farmacêuticas, Faculdade de Ciências Farmacêuticas de Ribeirão Preto, Universidade de São Paulo, Brasil, Avenida do Café, s/nº. - Campus Universitário da USP - Ribeirão Preto - SP - 14040-903 - Brasil. Telefone: 55 (16) 3602-4236. E-mail: andrebaldoni@ yahoo.com.br 
Casuistic and Methods. It was conducted a search through the crossing of the descriptor "Pharmacoepidemiology" with the selected country indexed in PubMed/MedLine. To search of the Brazilian articles in SciELO was used the descriptor "Farmacoepidemiologia". The research in both databases included all articles published until 06/18/2011, excluding review articles. Results and Discussion. It was found 620 articles indexed in PubMed/MedLine, with emphasis on the greater number of publications in the United States $(n=146)$ and Brazil ( $\mathrm{n}=14)$ among developed and developing countries selected, respectively. In relation to Brazilian studies, it was analyzed 48 articles indexed in PubMed/MedLine and SciELO. The Brazilian publications in the field of Pharmacoepidemiology started in 2001 and did not demonstrated pattern of evolution when the two databases was analyzed. The majority of the Brazilian studies were conducted in Southeast and South of Brazil $(n=40)$ and they used primary sources for data collection $(n=33)$. There were differences in the distribution of articles among the three areas of Pharmacoepidemiology, with a predominance of Drug Utilization Studies $(n=39)$. Conclusion. Despite the slight increase in the number of articles in the field of Pharmacoepidemiology in Brazil, there is a quantitative discrepancy when compared to those conducted in European and North America countries. In addition, it is necessary to develop pharmacoepidemiological studies in different populations and all regions of Brazil.

Keywords: Brazil. Drug Utilization Studies. Pharmacoepidemiology. Pharmacoeconomic. Pharmacovigilance.

\section{INTRODUÇÃO}

A Farmacoepidemiologia é definida como "aplicação do método e raciocínio epidemiológico no estudo dos efeitos benéficos e adversos - e do uso de medicamentos em populações humanas" (Brasil, 1998). O O termo Farmacoepidemiologia contém dois componentes: "fármaco" e "epidemiologia". Este campo de estudo faz a ponte entre duas grandes áreas: a farmacologia clínica, que estuda os efeitos dos fármacos em humanos, e a epidemiologia, que estuda a distribuição e os determinantes de doenças na população. Além disso, outras áreas do conhecimento são importantes para a realização dos estudos farmacoepidemiológicos como, por exemplo, a ciências sociais e a estatística (Strom, 2005).

A princípio, com o surgimento da Farmacoepidemiologia na década de 1960, esta ciência foi subdividida em duas grandes subáreas, sendo elas a Farmacovigilância e os Estudos de Utilização de Medicamentos (EUMs). Entretanto entre as décadas de 1990 e 2000 existiu a necessidade de estudos voltados para a aplicação da economia em saúde, surgindo a terceira vertente dos estudos farmacoepidemiológicos, a Farmacoeconomia (Strom, 2005).

De acordo com a Organização Mundial de Saúde (OMS) os EUMs são definidos como "estudos que compreendem a comercialização, distribuição, prescrição, dispensação e uso dos medicamentos em uma sociedade, com especial enfoque em suas 
conseqüências médico-sanitárias, sociais e econômicas" (OMS, 1977). Além disso, estes estudos apresentam uma visão global da utilização de medicamentos em uma sociedade, uma vez que podem descrever padrões de uso; constatar variações nos perfis terapêuticos no curso do tempo; avaliar os efeitos de medidas educativas, informativas, reguladoras; estimar o número de indivíduos expostos; detectar doses excessivas, uso inadequado, doses insuficientes e utilização indiscriminada; e estimar necessidades de medicamentos de uma sociedade (OMS, 2003; 2004a). Dessa forma, podem contribuir para a realização de intervenções adequadas e oportunas nos sistemas de saúde e na Gestão da Assistência Farmacêutica, promovendo o uso racional dos medicamentos (Guidoni et al., 2009).

A outra vertente, a Farmacovigilância, possui seus objetivos direcionados a questões de segurança no uso dos medicamentos, e é definida pela OMS (2004b) como a "ciência relacionada à detecção, avaliação, compreensão e prevenção dos efeitos adversos ou quaisquer problemas relacionados a medicamentos". Por exemplo, os estudos de Farmacovigilância podem contribuir fundamentalmente na identificação de novas restrições e para retirada de medicamentos do mercado, como recentemente ocorreu com a sibutramina e o rimonabanto.

Por fim, a Farmacoeconomia é uma subárea da economia em saúde e situa-se entre duas grandes áreas tradicionais, a saúde e a economia, podendo ser definida, segundo a International Society for Pharmacoeconomics and Outcomes Research (ISPOR) como "o campo de estudo que avalia o comportamento de indivíduos, empresas e mercados com relação ao uso de produtos, serviços e programas farmacêuticos, e que frequientemente enfoca os custos $\mathrm{e}$ as conseqüências desta utilização (Pashos et al., 1998). Dessa forma, é a aplicação da teoria econômica a farmacoterapia ou avaliação econômica do medicamento (Castilho, 1995).

As três vertentes dos estudos farmacoepidemiológicos são ferramentas primordiais para promoção do acesso e uso racional dos medicamentos pela população. Dessa forma, o presente estudo possui como objetivo estimar quantitativamente os artigos sobre Farmacoepidemiologia publicados mundialmente, além de analisar o perfil e a evolução dos estudos farmacoepidemiológicos no Brasil.

\section{CASUÍSTICA E MÉTODOS}

\subsection{Análise quantitativa dos artigos} publicados mundialmente sobre Farmacoepidemiologia 
Realizou-se uma busca no Pubmed/MedLine, utilizando-se o descritor "Phamacoepidemiology" [(NLM Controlled Vocabulary $(\mathrm{MeSH})]$ para análise quantitativa das publicações científicas mundiais referentes à Farmacoepidemiologia. Posteriormente, cruzou-se o descritor "Phamacoepidemiology" com os países de interesse selecionados "Argentina", "Australia”, "Brazil”, "Canada", "Chile", "China", "France", "Germany", "India", "Italy", "Japan", "Mexico", "Netherlands", "Portugal”, "Russia", "Spain", "Sweden", "Switzerland", "United Kingdom" e "United States".

Os principais países da América do Sul, América do Norte, Europa, Ásia e Oceania, selecionando desenvolvidos e emergentes, foram incluídos no estudo. Dessa forma, foi possível identificar o número total de artigos indexados no PubMed/MedLine e a quantidade de artigos por país de interesse até 18/06/2011, sendo excluídos os artigos de revisão.

\subsection{Análise dos artigos publicados no} Brasil sobre Farmacoepidemiologia

\section{O SciELO - A Scientific Electronic} Library Online - é uma biblioteca eletrônica que abrange uma coleção selecionada de periódicos científicos brasileiros
(http://www.SciELO.br/).

Para a análise dos artigos científicos publicados no Brasil, realizou-se busca nesta base de dados através do descritor "Farmacoepidemiologia" que foram publicados até 18/06/2011. Além disso, foram avaliados os estudos brasileiros encontrados no PubMed/MedLine, destacando-se que artigos contidos nas duas bases de dados foram considerados uma única vez.

Para esta seleção foram excluídos artigos de revisão, nota, atenção farmacêutica, descrição de serviços ou implantação de estratégias em saúde.

A análise do perfil e evolução dos estudos farmacoepidemiológicos no Brasil foi realizada através dos resumos dos artigos identificados no PubMed/MedLine e SciELO e, quando este não possuía as variáveis de interesse, realizou-se análise completa do artigo científico. Os autores consideraram variáveis de interesse o ano de publicação, região do país onde foi realizado o estudo, identificação das fontes de obtenção dos dados, objetivo principal e o tamanho da amostral do estudo.

Os autores consideraram como fontes de informações primárias os formulários e questionários, e como secundárias as prescrições, notificações de receitas, prontuários e bancos de dados eletrônicos. Para a classificação dos estudos 
farmacoepidemiológicos de acordo com as vertentes EUMs, Farmacovigilância e Farmacoeconomia, foi realizado a análise do objetivo principal dos estudos selecionados.

\section{RESULTADOS}

Foram encontrados 981 artigos científicos com o descritor "Pharmacoepidemiology" na base de dados PubMed/MedLine, sendo 139 de revisão. Do total, 724 foram publicados pelos 20 países selecionados pelos autores do presente estudo, porém 104 foram excluídos por se tratarem de artigos de revisão, totalizando 620 artigos incluídos na análise quantitativa do estudo (Tabela 1).

Tabela 1- Número de artigos científicos indexados no PubMed/MedLine até 18/06/2011 com o descritor "Pharmacoepidemiology" e país correspondente $(n=620)$.

\begin{tabular}{cccc}
\hline País & Artigos (n) & País & Artigos (n) \\
\hline Estados Unidos & 146 & Brasil & 14 \\
Holanda & 67 & Japão & 13 \\
França & 65 & Austrália & 11 \\
Canadá & 53 & Índia & 08 \\
Reino Unido & 53 & Portugal & 06 \\
Itália & 47 & México & 04 \\
Alemanha & 42 & China & 04 \\
Suécia & 41 & Rússia & 03 \\
Espanha & 24 & Argentina & 00 \\
Suíça & 19 & Chile & 00 \\
\hline
\end{tabular}

Fonte: Os autores. responsáveis pela maior produção de artigos científicos nesta área do conhecimento, destacando-se os Estados Unidos e dentre os países emergentes analisados, o Brasil demonstra maior produção científica nesta área (Tabela 1).

Quando considerado os artigos realizados no Brasil disponíveis no PubMed/MedLine e SciELO, foram encontradas 67 publicações, sendo que 19 foram desconsideradas da análise final por se enquadrarem nos critérios de exclusão do estudo, totalizando 48 artigos incluídos.

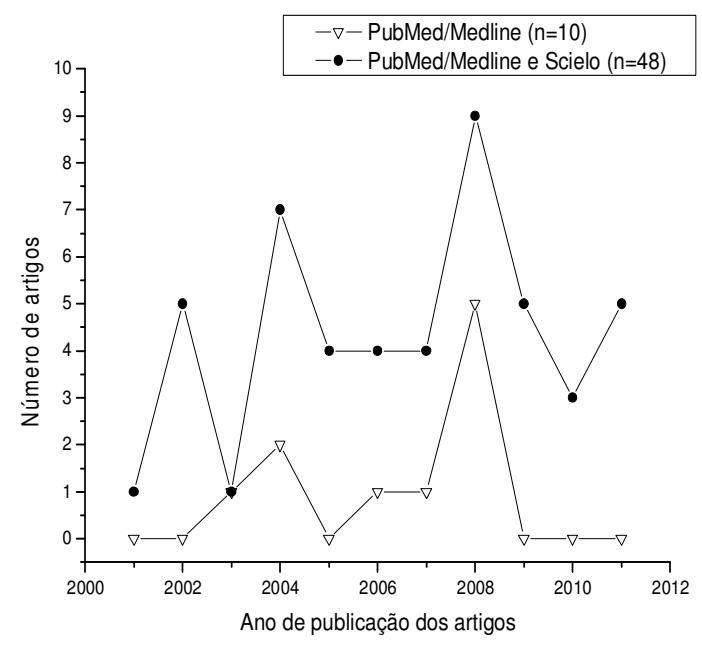

Figura 1- Número de artigos brasileiros sobre Farmacoepidemiologia indexados no PubMed/MedLine e SciELO até 18/06/2011 versus ano de publicação $(n=48)$.

Fonte: Os autores.

Os países desenvolvidos são 
Com relação ao ano de publicação dos artigos identificados no Brasil, foi possível observar que as publicações iniciaram no ano de 2001 e não apresentaram padrão de evolução nas duas bases de dados analisadas (Figura 1). Além disso, pode-se destacar que a maioria destes estudos concentra-se na região Sudeste e Sul do país (Tabela 2).

Tabela 2- Número de artigos sobre Farmacoepidemiologia indexados no PubMed/MedLine e SciELO até 18/06/2011 realizados nas diferentes regiões brasileiras $(n=48)$.

\begin{tabular}{cc}
\hline Região brasileira* & Artigos (n) \\
\hline Sudeste & 22 \\
Sul & 18 \\
Nordeste & 05 \\
Centro-Oeste & 02 \\
Norte & 00 \\
\hline
\end{tabular}

* Um estudo foi realizado em 27 capitais brasileiras.

Fonte: Os autores.

Considerando os 48 artigos selecionados, observa-se que 33 e 10 utilizaram, respectivamente, fontes primárias e secundárias de informação, quatro utilizaram os dois tipos de fontes para obtenção dos dados e um manuscrito não foi possível identificar a fonte de informação utilizada. Em adição, observa-se elevado número de estudos que utilizaram fontes primárias de informação com tamanho amostral superior 1000 indivíduos $(n=12)$

(Figura 2).

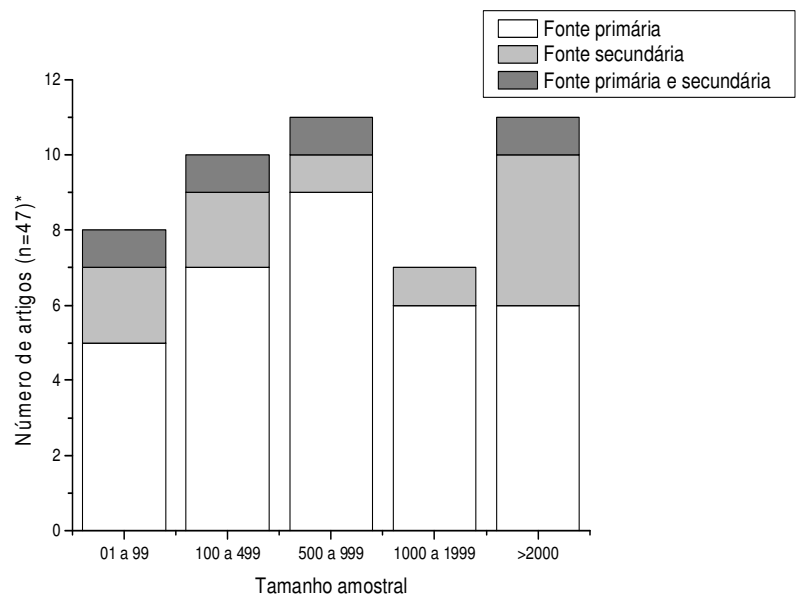

Figura 2- Distribuição dos artigos sobre Farmacoepidemiologia indexados no PubMed/MedLine e SciELO até 18/06/2011 de acordo com a fonte de informação e o tamanho amostral da população estudada $\left(\mathrm{n}=47^{*}\right)$.

* Não foi possível identificar a fonte de informação de um artigo selecionado. Fonte: Os autores.

Com relação à classificação dos estudos farmacoepidemiológicos, dentre as três vertentes da Farmacoepidemiologia, observa-se uma discrepância na distribuição dos artigos com predomínio dos EUMs (Tabela 3). Neste contexto, vale destacar que os estudos com a população idosa corresponderam a $60,0 \%$ dos 15 EUMs realizados com os grupos de pacientes especiais (crianças, gestantes, idosos). 
Tabela 3. Número de artigos sobre Farmacoepidemiologia indexados no PubMed/MedLine e SciELO até 18/06/2011 de acordo com a vertente dos estudos farmacoepidemiológicos $(n=48)$.

\begin{tabular}{|c|c|}
\hline $\begin{array}{c}\text { Vertentes da } \\
\text { Farmacoepidemiologia (n) }\end{array}$ & $\begin{array}{c}\text { Principal objetivo do estudo } \\
\text { (n) }\end{array}$ \\
\hline \multirow{5}{*}{$\begin{array}{l}\text { Estudo de Utilização de } \\
\text { Medicamentos (39) }\end{array}$} & Perfil utilização/consumo (27) \\
\hline & Perfil/análise prescrição (7) \\
\hline & $\begin{array}{l}\text { Conhecimento do usuário } \\
\text { sobre medicamentos (2) }\end{array}$ \\
\hline & Automedicação (2) \\
\hline & Polifarmácia (1) \\
\hline \multirow[t]{2}{*}{ Farmacovigilância (7) } & $\begin{array}{l}\text { Reação Adversa a } \\
\text { Medicamentos (4) }\end{array}$ \\
\hline & Interações Medicamentosas (3) \\
\hline Farmacoeconomia (2) & $\begin{array}{l}\text { Avaliar os gastos com } \\
\text { antimicrobianos (1) } \\
\text { Avaliar os gastos privados com } \\
\text { medicamentos (1) }\end{array}$ \\
\hline
\end{tabular}

Fonte: Os autores.

\section{Discussão}

Desde o surgimento da Farmacoepidemiologia (1960), várias iniciativas têm sido realizadas para a expansão desta área do conhecimento. Os países da Europa e da América do Norte foram os pioneiros em estudos farmacoepidemiológicos (Strom, 2005) e, até os dias atuais, são os países que apresentam a maior quantidade de artigos publicados sobre esta temática (Tabela 1). Este fato pode estar relacionado a diversos fatores como a maior organização na tecnologia da gestão e da utilização de medicamentos nestes países, o elevado rigor na regulamentação do mercado farmacêutico, o maior aporte financeiro às pesquisas científicas e o acesso a fontes de informações completas e de abrangência nacional.

Neste contexto, verifica-se que o número de artigos brasileiros encontra-se discrepantes quando comparados aos países mais desenvolvidos (grupo de países pertencentes ao G8). Entretanto, o Brasil ocupa posição de destaque nas publicações de artigos nesta área do conhecimento quando comparado aos países da América do Sul (Chile e Argentina), países emergentes como Rússia, Índia e China (que juntamente com o Brasil estão inseridos no grupo de países emergentes, o BRIC), e de alguns países considerados desenvolvidos (Japão, Austrália e Portugal) (Tabela 1). Dessa forma, qual postura o Brasil almeja adotar: manter a realidade atual ou ousar para se aproximar das grandes potências mundiais?

No Brasil, a Farmacoepidemiologia começa a ser discutida entre autoridades e profissionais da área de saúde nas décadas de 1970 e 1980. Porém, esse novo campo de estudo torna-se conhecido no contexto nacional a partir da década de 1990, com a criação da Sociedade Brasileira de Vigilância de Medicamentos (Sobravime, 1991) e dos Centros de Informação de Medicamentos (Castro, 1999), e posteriormente com a promulgação em 1998 da Política Nacional de Medicamentos (PNM) (Brasil, 1998), em 1999 da criação da Agência Nacional de 
Vigilância Sanitária (ANVISA) (Brasil, 1999), e em 2004 da Política Nacional de Assistência Farmacêutica (PNAF) (Brasil, 2004). Isto pode ser evidenciado pelos achados do presente estudo, no qual os primeiros artigos científicos sobre esta temática foram publicados no início do século XXI (Figura 1).

Por conseguinte, não existe um padrão característico de crescimento desejado no número de artigos científicos brasileiros sobre esta temática no país (Figura 1), pois não houve um crescimento linear durante os 10 anos de publicação. Dessa forma, espera-se que o número de artigos publicados seja progressivo. Além disso, verifica-se que nos últimos 30 meses nenhum artigo sobre Farmacoepidemiologia foi publicado em periódicos indexados no PubMed/MedLine, fazendo-se a necessidade de refletir sobre a visibilidade e qualidade dos estudos realizados no Brasil.

Com relação às localidades de realização dos estudos brasileiros (Tabela 2), é importante destacar que houve predominância de artigos originários das regiões Sudeste e Sul do país, as quais concentram elevado número de instituições de ensino superior e centros de pesquisas, maior produto interno bruto e agências estaduais de fomento com grande aporte financeiro. Por outro lado, visto que são estudos onerosos, é possível que esta predominância esteja relacionada com a concentração de pesquisadores nestas regiões?

Frente à concentração dos artigos em apenas duas regiões do país, verifica-se a necessidade de incentivar a realização destes estudos a nível nacional, principalmente devido à diversidade populacional brasileira e a interferência deste fator na tecnologia de utilização dos medicamentos.

Quando analisadas as fontes de informações para obtenção dos dados, os artigos avaliados utilizaram predominantemente fontes de informações primária. Tal fato pode estar relacionado com a baixa disponibilidade de fontes de informações secundárias, tais como banco de dados eletrônicos local e nacional. De acordo com o Guidelines for Good Pharmacoepidemiology Practices, a escolha da fonte de informação adequada é fundamental para a realização dos estudos farmacoepidemiológicos (Andrews et al., 2008), pois esta etapa poderá influenciar no tamanho amostral, variáveis a serem coletadas, tempo de coleta, planejamento econômico, confiabilidade dos dados coletados e aplicabilidade dos resultados obtidos (Witt, 1973; Cervo, Bervian, 1977; Schneeweiss, Avorn, 2005; Moreira, Caleffe, 2006; Harpe, 2009).

Apesar da relevância e aplicabilidade 
dos resultados oriundos de estudos realizados com fontes primárias, ressalta-se a importância de implementar banco de dados eletrônicos nos serviços de saúde com fins terapêuticos e administrativos. Em adição, isto permitiria a realização de estudos farmacoepidemiológicos rápidos, menos onerosos a longo prazo, de abrangência nacional e em número elevado de indivíduos, quando comparados as fontes primárias de informações. Tentativas recentes, mesmo que escassas, tentam suprimir tal deficiência, como a criação do Sistema Nacional de Gerenciamento de Produtos Controlados (SNGPC) e o Sistema Nacional de Gestão da Assistência Farmacêutica (HORUS).

Com relação às vertentes da Farmacoepidemiologia, observou-se carência de estudos sobre Farmacovigilância no Brasil (Tabela 3), a qual demonstra a necessidade de ações governamentais, políticas educacionais, sanitárias e capacitação dos profissionais de saúde. Estas ações devem ser direcionadas para a melhoria na segurança da utilização dos medicamentos, uma vez que estes estudos complementam as informações dos ensaios clínicos controlados no período póscomercialização (Fautrel, 2004).

Corroborando com isto, o presente estudo demonstrou poucas publicações relacionadas à vertente da Farmacoeconomia (Tabela 3). Deve-se considerar que estes estudos são fundamentais para auxiliar na racionalização dos investimentos em saúde no Brasil, uma vez que no período de 2002 a 2006, os gastos com insumos farmacêuticos tiveram incremento de $123,9 \%$, enquanto que os investimentos totais destinados à saúde aumentaram em 9,6\% (Brasil, 2007).

Por conseguinte, após a obtenção dos resultados do presente estudo, verifica-se a necessidade de realização de trabalhos que comparem a qualidade dos estudos farmacoepidemiológicos brasileiros com os realizados internacionalmente.

Além disso, deve ser considerado a aplicabilidade dos resultados obtidos nestes estudos para implementação de estratégias efetivas no sistema de saúde com a finalidade de melhorar a tecnologia de gestão e de utilização dos medicamentos.

\section{CONCLUSÃO}

Apesar do discreto crescimento no número de artigos sobre Farmacoepidemiologia no Brasil, existe uma discrepância quantitativa quando comparado aos realizados nos países da Europa e América do Norte. Além disso, verifica-se a necessidade da realização de estudos farmacoepidemiológicos de abrangência nacional, com maiores tamanhos amostrais e em diferentes grupos populacionais. 


\section{REFERÊNCIAS}

ANDREWS EB, ARELLANO FM, AVORN J, BORTNICHAK EA, CHEN R, DAI WS, et al. Guidelines for good pharmacoepidemiology practices (GPP). Pharmacoepidemiol Drug Saf, , v.17, n. 2, p.200-8, 2008.

BRASIL. Portaria n. 3.916, de 30 de outubro de 1998. Dispõe sobre a Política Nacional de Medicamentos 1998. Diário Oficial da União, Brasília, DF; 1998.

BRASIL. Lei n. 9.782, de 26 de janeiro de 1999. Define o Sistema Nacional de Vigilância Sanitária, cria a Agência Nacional de Vigilância Sanitária, e dá outras providências. Diário Oficial da União, Brasília, DF; 1999.

BRASIL. Resolução no 338, de 06 de maio de 2004. Aprova Política Nacional de Assistência Farmacêutica. Diário Oficial da União, Brasília, DF; 2004.

BRASIL. Ministério da Saúde. Evolução dos Gastos do Ministério da Saúde com Medicamentos. Brasília: Editora do Ministério da Saúde; 2007. 34p.

CASTILHO JAS. Farmacoeconomía y evaluación econômica de medicamentos. Introdución. In: Castilho JAS, Llach XB, Forns J. Farmacoeconomia: evaluación econômica de medicamentos. Madrid: Editores médicos. 1995. p.19-30.

CASTRO LLC. Farmacoepidemiologia no Brasil: evolução e perspectivas. Ciênc. Saúde Coletiva, v. 4, n.2, p.405-10, 1999.

CERVO AL, BERVIAN PA. Metodologia científica. 2 a ed. ver. amp. São Paulo: McGrawhill; 1977.

FAUTREL B. Pharmacoepidemiology: lessons from real life. Joint Bone Spine, v. 71, n.3, p.175-7, 2004.

GUIDONI CM, OLIVERA CMX, FREITAS O, PEREIRA LRL. Diabetes Mellitus e Sistema Único de Saúde: Análise do Modelo Atual. Braz. J. Pharm. Sci., v. 45, n.1, p.3748, 2009.

HARPE SE. Using Secondary Data Sources for Pharmacoepidemiology and Outcomes Research. Pharmacotherapy, v.29, n.2, p. 138-53, 2009.

MOREIRA H, CALEFFE LG. Metodologia da pesquisa para o professor pesquisador. Rio de Janeiro: DP\&A; 2006.

OMS - Organização Mundial de Saúde . La seleción de medicamentos essenciales. Genebra, OMS, 6p, 1977.

OMS - Organização Mundial de Saúde. Introduction to drug utilization research. WHO International Working Centre for Drug Statistics Methodology, WHO Collaborating Centre for Drug Utilization Research and Clinical Pharmacological Services, 48p, 2003.

OMS - Organização Mundial de Saúde (OMS). How to investigate the use of medicines by consumers. Genebra: World Health Organization, 98p, 2004a.

OMS - Organização Mundial de Saúde. La farmacovigilancia: garantía de seguridad en el uso de los medicamentos: perspectivas políticas de la OMS sobre medicamentos. Genebra: World Health Organization, 7p, 2004b.

PASHOS CL, KLEIN EG, WANKE LA. ISPOR Lexicon. International for Pharmacoeconomic and Outcomes Research. Princeton; 1998.

SCHNEEWEISS S, AVORN J. A review of uses of health care utilization databases for 
epidemiologic research on therapeutics. J

Clin Epidemiol, v.58, n.4, p.323-37, 2005.

SOBRAVIME - Sociedade Brasileira de

Vigilância de Medicamentos. Estatutos da

Sociedade Brasileira de Vigilância de

Medicamentos. Boletim n.1, 1991.

STROM BL. What is

Pharmacoepidemiology? In: Strom BL, editor. Pharmacoepidemiology. 3rd ed.

Chichester (UK): JohnWiley \& Sons Ltd. p.3-

$15,2005$.

WITT A. Metodologia de pesquisa. São

Paulo: Resenha Tributária; 1973. 